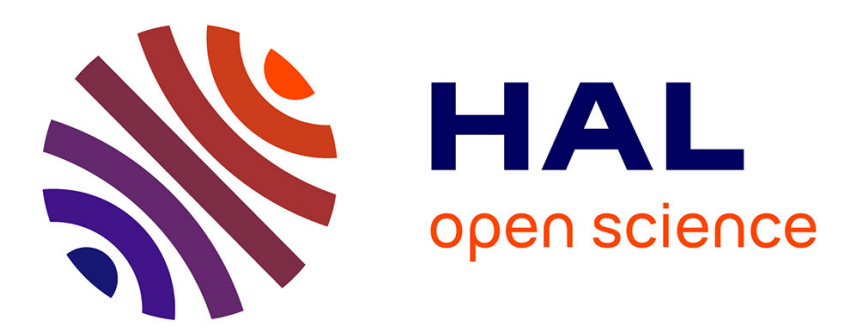

\title{
Remote Secure Decentralized Control Strategy for Mobile Robots
}

Philippe Fraisse, René Zapata, Walid Zarrad, David Andreu

\section{To cite this version:}

Philippe Fraisse, René Zapata, Walid Zarrad, David Andreu. Remote Secure Decentralized Control Strategy for Mobile Robots. Advanced Robotics, 2005, 19 (9), pp.1027-1040. 10.1163/156855305774307040 . lirmm-00105293

\section{HAL Id: lirmm-00105293 \\ https://hal-lirmm.ccsd.cnrs.fr/lirmm-00105293}

Submitted on 11 Oct 2006

HAL is a multi-disciplinary open access archive for the deposit and dissemination of scientific research documents, whether they are published or not. The documents may come from teaching and research institutions in France or abroad, or from public or private research centers.
L'archive ouverte pluridisciplinaire HAL, est destinée au dépôt et à la diffusion de documents scientifiques de niveau recherche, publiés ou non, émanant des établissements d'enseignement et de recherche français ou étrangers, des laboratoires publics ou privés. 


\title{
Remote Secure Decentralized Control Strategy for Mobile Robots
}

\author{
P. Fraisse, R. Zapata, W. Zarrad, D. Andreu \\ Université Montpellier II, LIRMM \\ 161 rue Ada, Montpellier, 34000 France
}

\begin{abstract}
The main goal of this paper is to define, study and analyse a remote control architecture for a set of non-holonomic robotic vehicles. This project gathers three laboratories and the French Army Research Office. Each of these laboratories deals with a part of this multidisciplinary project which includes coordinated control, control architecture, control with time delay and monitoring of the wireless network. In this paper, we propose to present the whole goal of this project including the basis experimental setup developed to validate our control algorithm. Secondly, we focuse on a new decentralized control strategy that uses the Leader-Follower principle. The originality of this paper stems from the use of the signal level of the wireless connection as a control vector. Indeed, each vehicle is fitted with two wireless devices. One of them is equipped of a sector antenna fitted on DC-motor to track the direction of better receiving level. Thus, it allows us to know the relative angular position of the Follower pointing out the Leader. By using the wireless technology as sensor instead of vision for instance, allows a longer distance of coordinated control loop between each vehicle (approx. 100m) even if the GPS information is not available.
\end{abstract}

\section{Introduction}

The control of multiple cooperative robotic vehicules is becoming an important robotics research field. A set of robotic vehicles can perform some tasks more efficiently than a single one. Missions such as exploration or rescue need to cover a large area. Sharing sensor information about their current and previous locations, the vehicles can also interact with the remote operator to concentrate the search around a given area. Other types of cooperative tasks have been addressed such as cooperative transport [1] or soccer playing [2]. To perform these tasks, different cooperative techniques from decentralized control to animal-like cooperative behavior have been studied. These techniques are based on different kind of sensors such as vision, position or velocity. The sharing of this information is often performed with wireless communications in the case of decentralized control.

Our research works focused on new control strategies of multiple vehicles based on GPS position and wireless communications. The wireless communications based on Wifi technology between each vehicle allow a geometric formation to be spread over a large spatial terrain (10 to $200 \mathrm{~m}$ between each vehicle). The vehicles or the formation will be teleoperated by a remote operator within the Wifi communication area. The objective is to analyse and perform a new control algorithm to coordinate robotic vehicles. This algorithm is based on centralized or decentralized control laws. The control variables are the position vector obtained by a GPS sensor fitted on each vehicle and the signal level of wireless communications (see figure 1).

The wireless communications are used to share information except if the GPS sensor information is not available or the Received Signal Level (RSL) tends to zero. In that case, the communication device becomes a sensor and the signal stemming from the neighbor vehicle allows the vehicle which lost GPS 


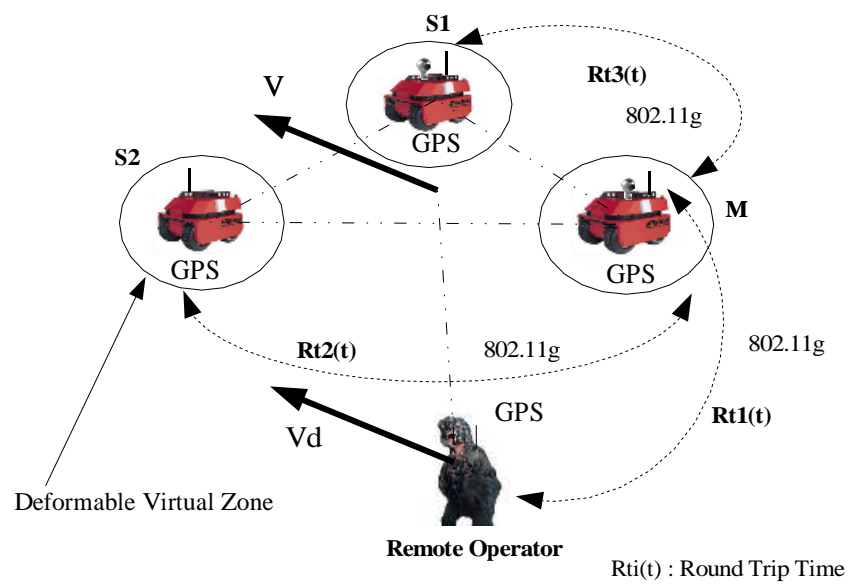

Figure 1: Cooperative robotic vehicles

data for instance, to stay in a communication zone. This control method secures the transmission links. It enables to spread over a mobile robot formation for a rescue mission in a safe manner.

\section{Control strategies}

We propose two control strategies (a decentralized one and a centralized one) dedicated for two different status of the formation:

- Decentralized control strategy will be based on a Leader-Followers structure as [3] and [4]. This control strategy will be chosen firstly when one robot is not in the communication area of the Operator at least. Secondly, when the GPS sensors information is partially available. Partially available means that the nearest vehicle to the vehicle without position information must have its GPS position information available.

- Centralized control strategy will be performed when all the vehicles will be in the communication area of the Operator and the GPS sensors information will be available.

In both cases, an obstacle avoidance algorithm, based on the Deformable Virtual Zone method (DVZ) and using ultrasound information [6], will be implemented on each vehicle and will be used as a reactive behavior control algorithm when unpredictable obstacles appear.

\subsection{Decentralized control law}

The decentralized control law is based on a Leader-Followers model (cf. figure 2). At the beginning of the experimentation, one of the robots which is in the communication area of the Operator, is arbitrary chosen as the Leader. The others are the Followers while assuming each robot is in a communication area of its nearest neighbor at least. Thus, if the position information given by the Leader's GPS is not available then the Leader assignment is automatically swapped to a vehicle which has a valid position.

The desired position of the Leader is defined by the current position of the operator and a distance $d_{o l}$ which could be constant or variable. The Follower desired positions are defined by the position of its nearest neighbor (Follower or Leader) rose by a distance $d_{f i_{f j}}$ (distance between Followers $i$ and $j$ ) or $d_{l_{f i}}$ (distance between the Leader and Follower $i$ ). Thus, the movement of the operator is automatically 


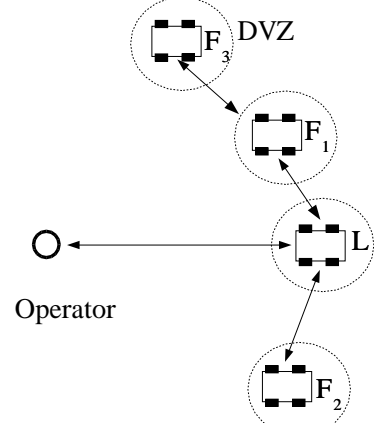

Figure 2: Decentralized control strategy

followed by the formation. In that strategy case, each Follower is also a Leader for its nearest neighbor. Meanwhile, the operator could receive some information from the Leader about the sensors fitted on each vehicle (ex.: video).

\subsection{Centralized control law}

The centralized control law is located in the near operator environment and broadcasts the displacement vector (velocity and steering angle) to the vehicles.

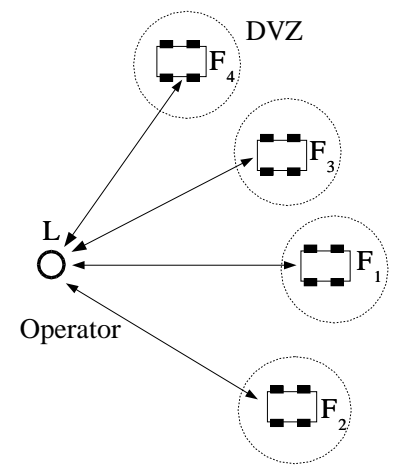

Figure 3: Centralized control strategy

In this case the Operator becomes the Leader and the motion of each vehicle depends on the Operator position and a constant or variable distance $d_{o_{f i}}$ between the Operator and the $i^{\text {th }}$ Follower. The desired position of the formation is defined by the current position of the Operator and some geometric constraints to hold the formation.

\subsection{Position/(Received Signal Level) hybrid control strategy}

Position/(Received Signal Level) hybrid control law is defined as a complementary control. Indeed, the signal level control loop is an external loop which provides an additional relative desired position $\Delta P_{d}$ to the absolute desired position $P$. Position and Received Signal Level vectors can be expressed as $P=\left[\begin{array}{lll}x & y & \theta\end{array}\right]^{t}$ and $R=\left[\begin{array}{ll}\lambda & l\end{array}\right]^{t}$, with $x$ and $y$ the cartesian position, $\theta$ the steering angle, $\lambda$ the angle between the directive antenna and vehicle and $l$ the level of the signal. To track the trajectory of its 
neighbor by using reception signal level and meanwhile, transmit its position to the second neigbhor (cf. figure 4), each vehicle must host two Wifi cards.

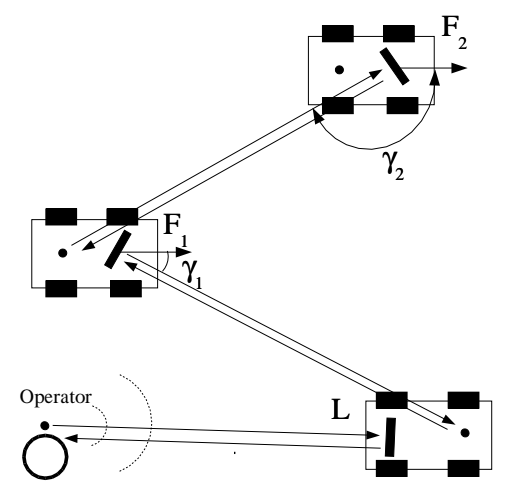

Figure 4: Decentralized hybrid control strategy

The first one drives a wireless unit using an omnidirectional antenna while the second one drives a sector antenna fitted on a DC-motor in order to follow the movement of its nearest neighbor. Thus, the angle $\lambda$ between two vehicles is given by the position sensor of the sector antenna DC-motor. The sector antenna is useful to emit data on the upload link in either case (decentralized or centralized control strategies). The download transmission will be performed via the card equipped with the omnidirectionnal antenna. Figures 2 and 3 illustrate these transmission links between the vehicles and Operator.

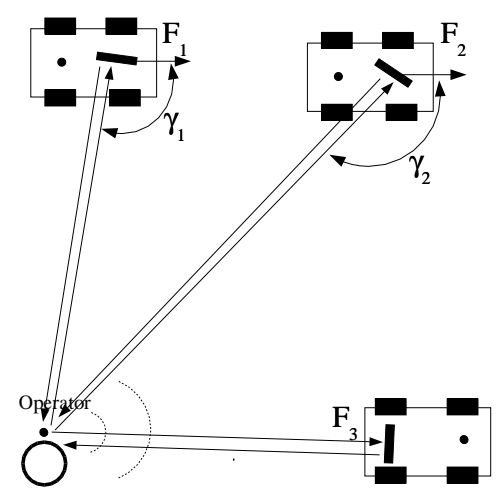

Figure 5: Centralized hybrid control strategy

The reception signal level control strategy holds the communication network available within the formation.

\section{Decentralized Control Algorithm}

\subsection{Leader-Follower Positioning}

Under decentralized control strategy, the set of coordinated mobile robots follows a Leader-Follower model (cf. figure 6). Each Follower deduces his position vector by using both GPS sensor and the angular position $\psi$ of the Wifi sector antenna. 


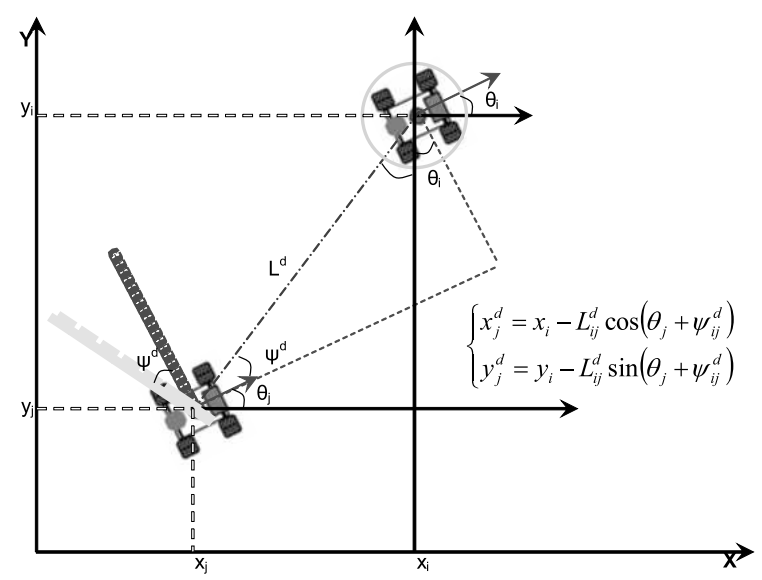

Figure 6: Decentralized

The Wifi motorized sector antenna gives the steering angle of the best receiving level from the Leader. Using this information and the absolute position given by the GPS sensor, we can define the expression of the desired position vector of the vehicle $j$ as :

$$
\begin{aligned}
& x_{j}^{d}=x_{i}-L_{i j}^{d} \cos \left(\theta_{j}+\psi_{i j}\right) \\
& y_{j}^{d}=y_{i}-L_{i j}^{d} \sin \left(\theta_{j}+\psi_{i j}\right) \\
& \theta_{j}^{d}=\operatorname{atan}\left(\frac{y_{j}^{d}-y_{j}}{x_{i}^{d}-x_{i}}\right)
\end{aligned}
$$

where $L_{i j}^{d}$ is the desired length between the vehicles $i$ and $j, \psi$ the angular position between the two vehicles and $\theta$ the relative orientation of the vehicle. The desired position of the First Leader is defined by the position of the Operator. The non-holonomy constraint can be written as :

$$
\begin{aligned}
& \dot{x}_{j}=v_{j} \cos \left(\theta_{j}\right) \\
& \dot{y}_{j}=v_{j} \sin \left(\theta_{j}\right) \\
& \dot{\theta}_{j}=\omega_{j}
\end{aligned}
$$

where $\omega_{i}$ is the steering angular velocity of the vehicle $i$ and $v_{i}$ its cartesian velocity. We can therefore write the direct kinematic equation as :

$$
\left(\begin{array}{c}
\dot{x}_{j} \\
\dot{y}_{j} \\
\dot{\theta}_{j}
\end{array}\right)=\left[\begin{array}{cc}
\cos \left(\theta_{j}\right) & 0 \\
\sin \left(\theta_{j}\right) & 0 \\
0 & 1
\end{array}\right]\left(\begin{array}{c}
v_{j} \\
\omega_{j}
\end{array}\right)=J\left(\begin{array}{c}
v_{j} \\
\omega_{j}
\end{array}\right)
$$

\subsection{Decentralized control algorithm}

At this step, we do not include the time delay constraint. We provide a mathematical task description in the decentralized control strategy. The control vector can be defined as :

$$
\left(\begin{array}{c}
U_{\dot{x}_{j}} \\
U_{\dot{y}_{j}} \\
U_{\dot{\theta}_{j}}
\end{array}\right)=K_{p}\left(\begin{array}{c}
x_{j}^{d}-x_{j} \\
y_{j}^{d}-y_{j} \\
\theta_{j}^{d}-\theta_{j}
\end{array}\right)
$$

The velocity control vector applied to the actuators can be defined as : 


$$
\mathbf{U}_{p_{j}}=\left(\begin{array}{c}
U_{v_{j}} \\
U_{\omega_{j}}
\end{array}\right)=J^{+}\left(\begin{array}{c}
U_{\dot{x}_{j}} \\
U_{\dot{y}_{j}} \\
U_{\dot{\theta}_{j}}
\end{array}\right)
$$

$J^{+}$is the pseudo-inverse of the jacobian defined by the direct kinematic equation 3 .

\subsection{Leader-Follower Positioning without GPS information}

Since, the GPS information for each vehicle is not always available, for instance within an Urban area, the decentralized control strategy could lost some vehicles. Each vehicle being a data communication relay between two other robots, the loss of one vehicle breaks down the chain of the coordinated control. To face up to this problem, we propose a new definition of the control vector $\mathbf{U}$ whose the components use the Received Signal Level (RSL). We propose:

$$
\mathbf{U}_{r_{j}}=\left(\begin{array}{c}
U_{v_{j}} \\
U_{\omega_{j}}
\end{array}\right)=\left(\begin{array}{l}
K_{p j}\left(R_{i j}^{d}-R_{i j}\right)+K_{v j}\left(\dot{R}_{i j}^{d}-\dot{R}_{i j}\right) \\
K_{p \omega j}\left(\theta_{j}^{d}-\psi_{j}\right)
\end{array}\right)
$$

where $R_{i j}^{d}$ the Received Signal Level of the vehicle $j$ from vehicle $i$.

\subsection{Global decentralized control algorithm}

The decentralized control algorithm without GPS information is based on the tracking of the transmission signal of the Leader. We assume that the angle $\psi$ of the motorized antenna measuring the best receiving level points out the nearest Leader. At this step of the study, we do not include the spatial propagation model of the signal. To switch the control law strategy from the position loop to the RSL loop, we insert a switching matrix $\mathbf{S}$ as :

$$
\mathbf{U}=\mathbf{S U}_{\mathbf{p j}}+(\mathbf{1}-\mathbf{S}) \mathbf{U}_{\mathbf{r j}}
$$

The control vector $\mathbf{U}=\left[U_{v_{j}} U_{\omega_{j}}\right]$ enables to connect either the position control loop or RSL control loop. The RSL control strategy allows to hold the vehicles gathered around the First Leader.

The switching between the Position and the Signal Level loops can be made automatically via the switching matrix $\mathbf{S}$ as soon as the GPS information disappears. Nevertheless, the Operator can also opt for this control mode to ensure a high quality of the transmission channel between the vehicles.

\subsection{Global decentralized control law including obstacle avoidance algorithm}

\subsubsection{DVZ definition}

The reactive control algorithm we use, is based on the definition of a deformable zone surrounding and protecting the robot. For mobile robots, this DVZ (Deformable Virtual Zone) is parameterized by the motion variables of the moving robot and can deform in the presence of distance information in the robot workspace (this information, denoted $I$, is obtained by ultrasound sensors). When obstacles enter the sensor space, they induce deformations of the DVZ that will be compensated by the robot motion controller. Therefore, the algorithm is a kind of 2-player game: the first one, e.g. the environment, induces undesired deformations and the second one, e.g. the robot controller, tries to rebuild the DVZ. This deformation can be written:

$$
\Delta=\Xi-\Xi_{h}=\beta(I)-\rho\left(\mathbf{U}_{\mathbf{d v z}}\right)
$$


where $\Xi=\beta(I)$ depends on the proximity information $I$, and $\Xi_{h}=\rho\left(\mathbf{U}_{\mathbf{d v z}}\right)$ represents the part of the DVZ controlled by the robot.

By differentiating equations (8) with respect to time, we get:

$$
\dot{\Delta}=-\nabla_{\pi}[\rho] \phi+\nabla_{I}[\beta] \psi
$$

where $\nabla_{\xi}[$.$] is the derivation operator with respect to the vectorial variable \xi$ and $\psi=\dot{I}$ and:

$$
\phi=\dot{\mathbf{U}}_{\mathbf{d v z}}=\left(\begin{array}{c}
\dot{U}_{v_{j}} \\
\dot{U}_{\omega_{j}}
\end{array}\right)
$$

This equation can be rewritten as:

$$
\dot{\Delta}=A \phi+B \psi
$$

Variations in $\Delta$ are controlled by a 2-fold input vector $u=\left[\begin{array}{ll}\phi & \psi\end{array}\right]^{T}$. The first control vector $\phi$, due to the robot controller, tends to minimize deformation of the DVZ. The second one, $\psi$, is unknown and induced by the environment itself.

\subsubsection{Control law}

Once equation (11) is obtained, its integration (i.e. the obtention of a "good" control vector $\phi$ ) can be computed in 3 steps:

1. Computing the intrusion of information field $I$ by measuring distance information in the $s$ directions of proximity sensors

2. Choosing the desired variation of this deformation as a function of the real deformation and its derivative:

$$
\dot{\Delta}_{\text {des }}=-K_{\text {prop }} \Delta-K_{\text {der }} \dot{\Delta}
$$

where $K_{\text {prop }}$ and $K_{d e r}$ are heuristically chosen.

3. Computing the best control vector $\check{\phi}$ at time $t$ obtained by inverting equation (11) after replacing the deformation derivative by its desired value $\dot{\Delta}_{\text {des }}$ :

$$
\check{\phi}=A^{\dagger}\left(\dot{\Delta}_{\text {des }}-B \hat{\psi}\right)
$$

where $A^{\dagger}$ is the inverse function (pseudo-inverse) of the linear function $A$ and $B \hat{\psi}$ is an estimation of the second control vector $\psi$ at time $t$ obtained at time $t-1$ :

$$
B \hat{\psi}(t)=\dot{\Delta}_{\text {measured }}(t-1)-A \phi(t-1)
$$

4. Integration of $\check{\phi}$ in order to obtain the control vector: $\mathbf{U}_{\mathbf{d v z}}=\int_{\mathbf{t}}^{\mathbf{t}+\mathbf{d t}} \check{\phi} \mathbf{d t}$

\subsubsection{Inclusion of the DVZ control law in the global decentralized control law}

When one of the robots meets an obstacle, the DVZ control law has to be applied in priority. This can be done by introducing a second switching matrix $\mathbf{S}_{\mathbf{d v z}}$. Now, equation (15) can be written:

$$
\mathbf{U}=\left(\mathbf{1}-\mathbf{S}_{\mathbf{d v z}}\right) \mathbf{S} \mathbf{U}_{\mathbf{p j}}+\left(\mathbf{1}-\mathbf{S}_{\mathbf{d v z}}\right)(\mathbf{1}-\mathbf{S}) \mathbf{U}_{\mathbf{r j}}+\mathbf{S}_{\mathbf{d v z}} \mathbf{U}_{\mathbf{d v z}}
$$




\section{Simulation results}

We have developed a simulator with the Graphical User Interface Tool of $M A T L A B^{13}$ which includes up to five non-holomic mobile robots with ultrasound sensors. The dynamic behavior of the vehicles is modelled and implemented within the software.

\subsection{Position/RSL control law with three vehicles}

The movement of the formation starts with the two followers controlled by the position of the Leader (see figure 7). At the time $t=36 s$ the matrix $\mathbf{S}_{2}$ of the Follower 2 switchs to the RSL loop with a desired Received Signal Level as : $R_{2}^{d}=-5 d B m$.

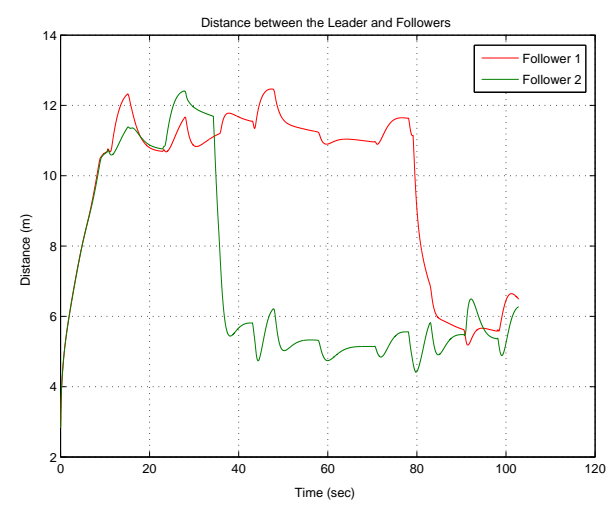

Figure 7: Distances between the Leader and Followers

At the time $t=80 \mathrm{~s}$, the Follower 1 switchs also to the RSL loop with $R_{2}^{d}=-5 \mathrm{dBm}$. The distances between the Leader and Followers are presented figure 7. The important tracking error between the desired and current distance for a couple of Leader-Follower is produced by the use of proportionnal gain. This compliance enables to avoid the blocking of the Followers when the Leader carries out a U-turn, for instance. Indeed, in that case the velocity needed to maintain a small tracking error is over the maximum velocity of the vehicle. The whole trajectory of the three robots is presented figure 8 .

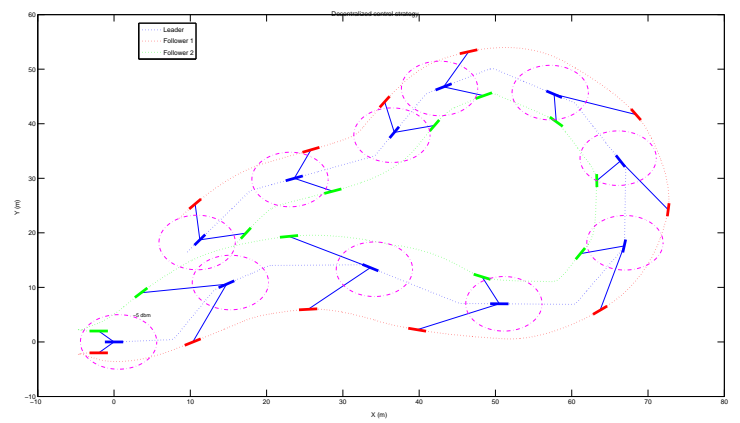

Figure 8: Cooperative Motion with three vehicles 


\subsection{Position/RSL control law with five vehicles}

The movement of the formation starts with five vehicles controlled by the position of the nearest Leader. In this case, the Followers 1 and 2 are respectively the Leaders of the Followers 3 and 4 . At the time $\mathrm{t}=80 \mathrm{~s}$ the Followers 1 and 3 switch to the RSL control law whith the desired RSL as $R_{1}^{d}=R_{3}^{d}=-25 \mathrm{dBm}$. The next switch of the control law is performed by the Follower 2 and 4 to the RSL at the time $\mathrm{t}=180 \mathrm{~s}$ with $R_{2}^{d}=R_{4}^{d}=-25 \mathrm{dBm}$.

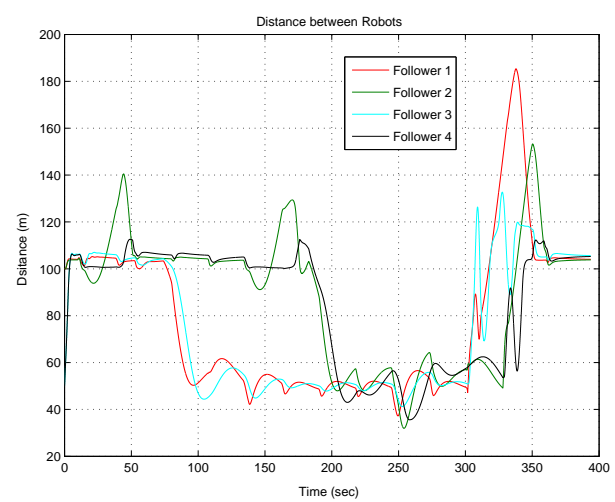

Figure 9: Distances between the Leaders and Followers

The tracking error between the two couples of 1-3 and 2-4 vehicles is higher than the three vehicles case. The maximum velocity requires to track the main Leader increases by adding new vehicles indeed. We migth modelize this formation by a set of Mass-Stiffness for each vehicle linked with its neigbhor in the both cases (Position and RSL control law). Nevertheless, the stability of the formation should be studied to find the maximum gain value which can be used to reduce the tracking error while keeping the stability. However, the maximum velocity constraint of each vehicle must be included in this study in order to obtain a realistic stability limit. The next step of this work will be dedicated to the study of the stability. The cooperative motion with five vehicles is presented figure 10.

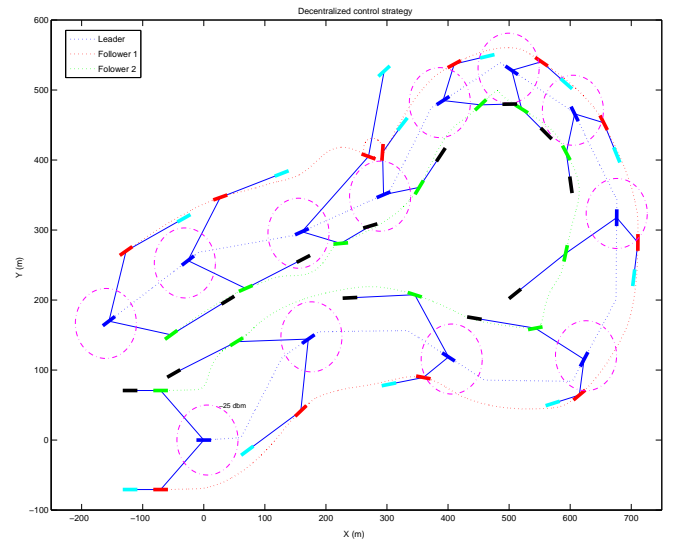

Figure 10: Cooperative motion with five vehicles 


\section{Conclusion}

This multidisciplinary research collaboration proposes a new strategy to control a set of coordinated vehicle based on GPS and received signal level sensors. The sensor of a reception signal level is a wireless communication device based on Wifi standard [8]. This strategy allows the communication link between robots to be maintain. Thus, the mobile robots are reachable even if the GPS sensors do not work. In this case, the geometric formation will not match with the initial one. That the condition to keep the vehicle in a communication area. We hope to test and validate our developments on the experimental setup as soon as possible in order to highlight the advantages and drawback of this method. This method will allow to spread over a large urban area, a set of mobile vehicles as a mobile communication network.

\section{Acknowledgement}

To deal with these different points, we created in September 2003, a multi-laboratories research national project (CNRS) with the support of the french army research office (DGA). This project is funded for 2 years. We are designing an experimental setup which is expected to run to early September 2004 .

\section{References}

[1] K. Kosuge, T. Oosumi, M. Satou, K. Chiba, K. Takeo,"Transportation of a single object by two decentralized-controlled nonholonomic mobile robots", in Proc. Conf. Robotics and Automation, Leuven, Belgium, May 1998, pp. 2989-2994.

[2] T. Weigel, J. Gutmann, M. Dietl, A. Kleiner, B. Nebel, "CS Freiburg : Coordinating Robots for Successful Soccer Playing, IEEE Transactions on Robotics and Automation, Special Issue on Advances in Multirobot Systems, Vol. 18, N 5, October 2002, pp. 685-699.

[3] J.T. Feddela, C. Lewis, D.A. Schoenwald, "Decentralized Control of Cooperative Robotic Vehicles : Theory and Application", IEEE Transactions on Robotics and Automation, Special Issue on Advances in Multirobot Systems, Vol. 18, $\mathrm{N}^{\circ}$ 5, October 2002, pp. 852-863.

[4] A.K. Das, R. Fierro, V. Kumar, J. P. Ostrowski, J. Speltzer, C. J. Taylor, "A Vision-Based Formation Control Framework", IEEE Transactions on Robotics and Automation, Special Issue on Advances in Multirobot Systems, Vol. 18, N 5, October 2002, pp. 813-825.

[5] P. Fraisse, A. Lelevé, "Teleoperation over IP network : Network Delay Regulation and Adaptive Control", Journal of Autonomous Robots, vol 15, $\mathrm{N}^{\circ}$ 3, November 2003, pp.225-235, ISSN: 09295593 .

[6] A. Cacitti, R. Zapata,"Reactive Behaviours of Mobile Manipulators Based on the DVZ Approach", IEEE Conference on Roborics and Automation, 2001, pp. 680-685.

[7] K. Goldberg and R. Siegwart, "Beyond Webcams: an Introduction to Online Robots", MIT Press, 2002.

[8] ANSI/IEEE Std.802.11, 1999 Edition, Part 11: "Wireless LAN Medium Access Control (MAC) and Physical Layer (PHY) Specifications. 